\title{
Tuning a mid-range rural WiFi-based mesh network
}

Carlos Rey-Moreno

William D. Tucker

Javier Simo-Reigadas

\begin{abstract}
Little is known about how mid-range, or several $\mathrm{km}$, distances affect multipoint-to-multipoint links when single radio nodes with omnidirectional antennas are used. In this poster, a real network with these characteristics is described and empirical results of its performance are presented. In particular, the effect of increasing the SlotTime value for optimizing the aggregated throughput has been analyzed. Results show the need to increase the SlotTime to considerably bigger values than those oberved for point-to-point and point-to-multipoint long-range links. With the new value of SlotTime, an increase of $115 \%$ in aggregated throughput was obtained over standard Slottime value.
\end{abstract}

\section{Categories and subject descriptors}

C.2.1 [Network Architecture and Design]:

Network Topology - Wireless Communication

\section{General terms}

Telecommunications, Design, Optimization.

\section{Introduction}

One of the key factors determining an architecture is the distance among the nodes forming it. There is a large body of work on long-range wireless mesh networks $[4,3]$; long- range being understood as tens of kilometers $(\mathrm{km})$. In most of those interpretations, long-range comes together with directional antennas due to the link budget and the power limitations in non-licensed bands. With directional antennas, static mesh networks are only feasible if each mesh node has several radios installed for connecting to several neighbors. Thus, limitations on available non-overlapping channels, number of radios, and power consumption strongly limit possible topologies.

Other approaches consider that a mesh node is a low-cost low-power node with only one radio [1]. Hence the whole mesh network operates on a single channel and performance is sacrificed because each node acts as a source/sink/router and forwarding packets over a single wireless interface halves the performance. Other effects reduce the performance even more. On the other hand, the gains include being cheaper, easier to install and maintain, more flexible, and valid when the capacity is still enough for actual applications. 


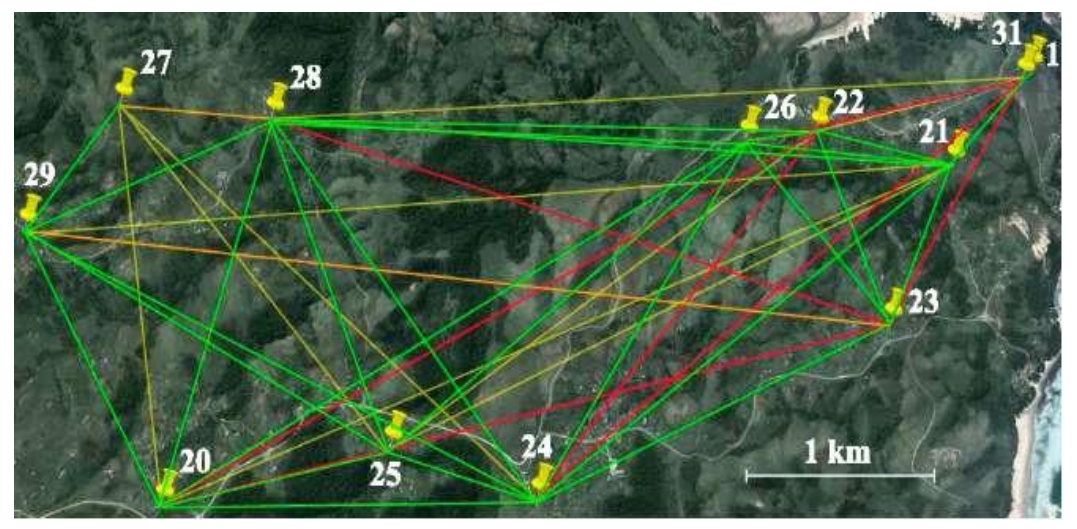

\section{Figure 1: Network links and distances}

From this second body of work, only [2] works on the range of few km (with a link up to $5 \mathrm{~km}$ ). However, in [2] there is no reference to the effect that distance over the standard limits may have played upon the performance of the links.

To the best of our knowledge the work presented here is the first one optimizing the performance of a real single radio mid-range multi-hop wireless mesh network. The mesh networks proposed here are not intended to reach regional scalability, but rather to provide sufficient fixed points of access (as many as nodes form the mesh backbone) to individual rural villages that will then be linked up to the Internet and/or together otherwise. These fixed points of access forming the mesh backbone could additionally provide the functionality of wireless access points and so allow for more wireless clients. Eventually, the mesh network must scale to provide blanket coverage for the entire village.

\section{Materials and methods}

The network consists of 12 nodes scattered around $30 \mathrm{~km}^{2}$. The connectivity graph of the network is shown in Figure 1, where nodes appear labelled by its IP last octect. The colors correspond to the following RSSI on both sides of the link: green for values above $12 \mathrm{dBm}$, orange for values between 9 and $12 \mathrm{dBm}$, and red when one or both are below $9 \mathrm{dBm}$. In yellow are links so weak that they disappeared when setting the operating mode to 802.11g.

Ten of the 12 nodes are located in private houses chosen by the local authority. One of the other two nodes provides access to the server (.1) and the last one is a repeater connecting the latter to the rest of the network (.31). The repeater is an off-the-shelf Ubiquiti NanoStation. The rest of the nodes are first version mesh potatoes, MPo1, with an outdoor 8dBi omnidirectional antenna on the ten nodes deployed in private homesteads. All nodes have been flashed with version 1.1 of the Village Telco Small Enterprise and Campus Network (VT-SECN) firmware.

This poster intends to study the effect of modifying the value of the Slottime, which has been proven to be key in long-range PtP and PtMP 802.11 networks [4]. To do so, 
we fix several relevant parameters in the network to the following values: a) radios used 802.11g PHY; b) broadcast messages sent at $6 \mathrm{Mbps}$ (fixing mcast_rate to 600o); c) unicast messages were sent at the rate chosen by Minstrel, c) radios operated in basic mode, not using RTS/CTS, d) ACKTimeout fixed to $57^{\wedge} \mathrm{s}$ (round trip propagation delay for the longest link in the network: $5.05 \mathrm{Km}$ between 21 and 29); d) beacon interval set to 100ms and OGM interval to 1s (both default), e) Hop penalty to apply to multi-hop routes in badman-adv fixed set to 10 (default value). Slotime values from $9{ }^{\wedge} \mathrm{s}$ (the standard value for 802.11g) to $999^{\wedge} \mathrm{s}$ were tested by generating UDP traffic for 20 seconds in between two pair of nodes consecutively. Traffic generated was enough to saturate the link in between every pair of nodes.

\section{Results and discussion}

Figure 2 shows the aggregated end-to-end traffic between each pair of nodes in the network per value of SlotTime. Although this indicator does not show the maximum traffic the network can handle simultaneously, it offers a single figure that can be compared among different SlotTime values.

As can be seen in Figure 2, aggregated traffic peaks for SlotTime at $199^{\wedge} \mathrm{s}$, for which 92.75 Mbps are obtained. Although a single repetition was carried out for every SlotTime value, a similar trend is confirmed by the curve shown, and other values around the peak exhibit similar performance. These values contrast heavily with the value for SlotTime of $9^{\wedge} \mathrm{s}$ (standard for 802.11g), for which $43.11 \mathrm{Mbps}$ are obtained; and with the one using Madwifi's athctrl for the the longest link in the network (SlotTime, $24{ }^{\wedge} \mathrm{s}$ ) for which $74.87 \mathrm{Mbps}$ are obtained. The latter, shows a considerable improvement (57\%) over the standard value, but still, the maximum obtained fro our network is $23.9 \%$ higher.

Understanding the SlotTime as the time interval during which the transmission of a given node is vulnerable to collide with a transmission from a neighboring node, in point- to-point, and in point-to-multipoint networks, a relation between the value of SlotTime that optimizes the performance of the network and the distance of the longest link has been established theoretically as well as practically [4]. A tentative explanation for the behavior observed in this network may arise from the exposed node problem provided that the interference range for a node is much larger than its transmission/reception range. A node that receives signal under such sensitivity does not use the received information as a received packet, but rather that reception activates the CCA (clear channel assessment) and effectively freezes the contention window. Hence, there is a part of the MAC that should consider the interference range, instead of the transmission range, for the case of multi-hop networks, causing severe lose of transmission opportunities. Possibly, the longer the SlotTime is, the smaller the impact of the exposed-node problem results. However, the distance between the furthest nodes in the network able to cause this problem is not enough to justify the SlotTime values that maximizes traffic in this network. Thus, more research is needed to justify these positive empirically results. 


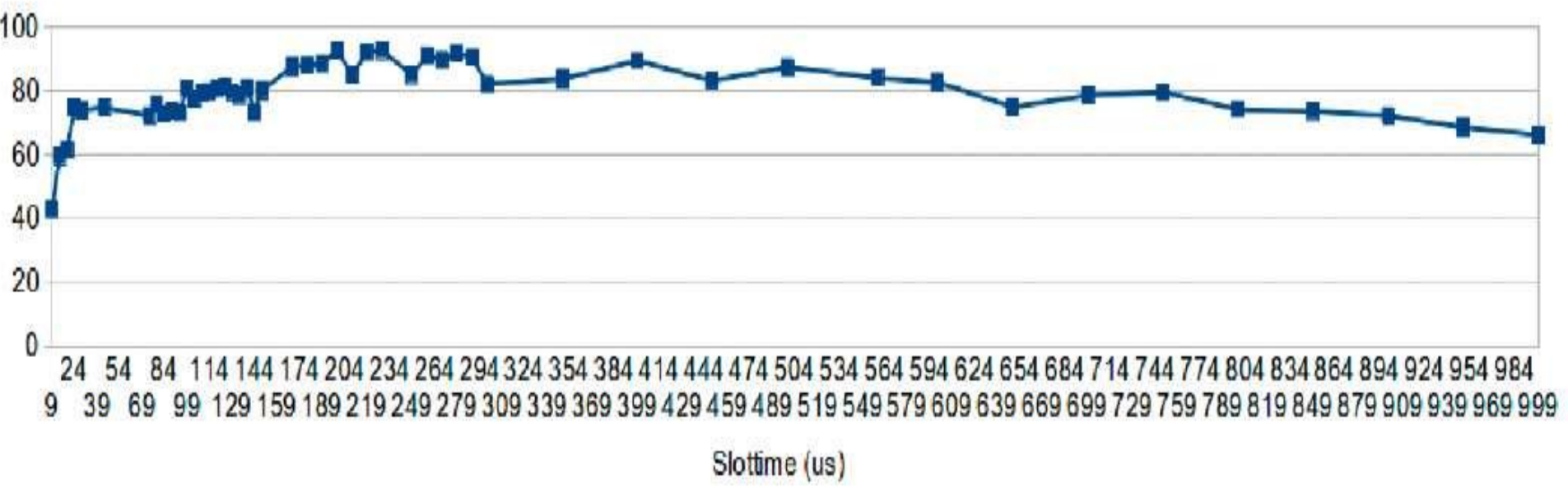

Figure 2: Variation of the aggregated throughput with SlotTime.

For the optimum Slottime value, end-to-end traffic has on average $1.40 \mathrm{Mbps}$, with the route .1 and .27 being the one carrying the least, $0.142 \mathrm{Mbps}$. These results are not comparable with much higher throughputs obtained in multi-radio mesh networks [4], but are enough for providing other services such as VoIP. Optimization of other parameters and introduction of new technologies could further increasaed the values obtained here.

\section{Acknowledgments}

We thank the Village Telco community, Thomas, Jay, Nic and Transcape. We also thank Telkom, Cisco, Aria Technologies and THRIP (Technology and Human Resources for Industry Partnership) for their financial support via the Telkom Center of Excellence (CoE). This work is based on the research supported in part by the National Research Foundation of South Africa (Grant number (UID) 75191). Any opinion findings and conclusion or recommendations expressed in this material are those of the authors and therefore the NFR does not accept any liability in this regard. 


\section{References}

[1] J. Bicket, D. Aguayo, S. Biswas, and R. Morris. Architecture and Evaluation of an Unplanned 802.11b Mesh Network. In MobiCom, pages 31-42, 2005.

[2] D. Johnson. Evaluation of a single radio rural mesh network in South Africa. In ICTD2007.

[3] B. Raman and K. Chebrolu. Experiences in Using WiFi for Rural Internet in India. IEEE Commun Mag, 45(1):104-110, 2007.

[4] J. Simo-Reigadas, A. Martinez-Fernandez, F.-J. Ramos-Lopez, and J. Seoane-Pascual. Modeling and Optimizing IEEE 802.11 DCF for Long-Distance Links. IEEE TMC, 9(6):881-896, 2010. 